\title{
Irreversible Permeability of Fe-Based Soft Magnetic Composites
}

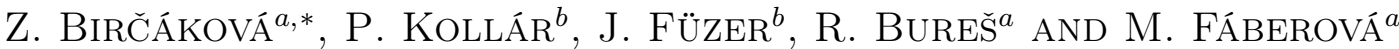 \\ ${ }^{a}$ Institute of Materials Research, Slovak Academy of Sciences, Watsonova 47, 04001 Košice, Slovakia \\ ${ }^{b}$ Institute of Physics, Faculty of Science, P.J. Šafárik University, Park Angelinum 9, 04154 Košice, Slovakia \\ The specification of the relation for irreversible relative permeability at initial magnetization curve is presented, \\ using the linear functions approximation of DC energy losses for Fe-resin soft magnetic composites. The exper- \\ imental and calculated dependencies of irreversible permeability vs. magnetic induction were compared, and \\ the empirical function in this relation was determined to be a constant. The proposed relation allows to cover \\ a wide induction range of validity of the linear function approximation (wider compared to the Steinmetz law \\ for the special case of Fe-resin composites), with even higher accuracy than the previous relation derived from \\ the Steinmetz law.
}

DOI: 10.12693/APhysPolA.137.843

PACS/topics: soft magnetic composite, irreversible permeability, DC energy losses

\section{Introduction}

Soft magnetic composites (SMCs) represent a remarkable kind of materials consisting of insulated ferromagnetic particles. Most significant advantages of SMCs over traditional soft magnetic materials are relatively low energy losses at medium to higher frequencies, relatively high saturation magnetization, and magnetic or thermal isotropy. The application range of SMCs covers various electromagnetic devices, and is still expanding [1-3]. Some magnetic quantities dependencies of SMCs are different from majority of bulk ferromagnets, as the magnetization process in SMCs is significantly influenced by the inner demagnetizing fields [3-5].

The magnetization reversal is realized by reversible and irreversible magnetization processes [6]. The proportions of these processes within the whole magnetization process determine the dependencies of magnetic quantities. Proportions of reversible processes can be found by the measurement of reversible permeability, which in practice is not very convenient [7-9]. Therefore, the relations enabling to obtain the reversible or irreversible permeability without the need of its measurement might be helpful.

In continuation to the previous work [10], the aim of this paper is to further specify the derivation of the relation for irreversible permeability at initial magnetization curve for Fe-resin SMCs with different Fe filler content and particle size distribution. The linear functions approximation of DC energy losses [11] is used. The calculated values are compared with experimental data.

*corresponding author; e-mail: zbircakova@saske.sk

\section{Experimental}

Fe-phenolphormaldehyde resin composites were prepared by conventional powder metallurgy methods in the form of a ring (outer diameter $24 \mathrm{~mm}$, inner diameter $18 \mathrm{~mm}$, height $2-3 \mathrm{~mm}$ ), with the different content of insulation (resin), and different particle size distribution $[9,10]$. Polycrystalline Fe powder ASC 100.29 (Höganäs $\mathrm{AB}$ Sweden [12]) was sieved obtaining particle size distributions with peaks at $45 \mu \mathrm{m}$ and $100 \mu \mathrm{m}$ (labelled SMC45 and SMC100), and homogenized with 5, 10, and 15 vol. \% (i.e., 1, 2, and 3 wt. \%) of phenolphormaldehyde resin (Bakelite ATM) and acetone. The compaction at uniaxial pressure $800 \mathrm{MPa}$ was performed, followed by the heat treatment at temperature $165^{\circ} \mathrm{C}$ for $1 \mathrm{~h}$ in electric furnace in air atmosphere $[9,10]$. Parameters of samples are given in Table I.

Initial magnetization curves and DC hysteresis loops were measured by DC fluxmeter-based hysteresisgraph with magnetic induction referred to the filler content of ferromagnetic material in sample. Total and differential relative permeability, DC energy losses and coercive field values were obtained. Reversible relative permeability at each point of initial curve was measured using the setup based on lock-in amplifier reading of

TABLE I

Parameters of SMC samples, where $x$ denotes ferromagnetic filler content.

\begin{tabular}{l|c|c|c}
\hline \hline \multicolumn{1}{c|}{ Sample } & $\begin{array}{c}\text { Fe powder } \\
\text { insulation } \\
\text { (vol.) }\end{array}$ & $\begin{array}{c}\text { Density } \\
{\left[\mathrm{g} / \mathrm{cm}^{3}\right]}\end{array}$ & $x[\%]$ \\
\hline SMC45-5\% & $95: 5$ & 6.60 & 83.4 \\
SMC45-10\% & $90: 10$ & 6.0 & 74.6 \\
SMC100-10\% & $90: 10$ & 6.05 & 75.2 \\
SMC45-15\% & $85: 15$ & 5.45 & 67.0
\end{tabular}


the induced voltage, in details described in $[9,10]$. The measurement principle was based on general definition for reversible relative permeability:

$$
\mu_{\mathrm{rev}}=\frac{1}{\mu_{0}} \lim _{\Delta H \rightarrow 0}\left(\frac{\Delta B}{\Delta H}\right)_{\left(H_{1}, B_{1}\right)},
$$

where $\mu_{0}$ is the magnetic constant, $\Delta H$ and $\Delta B$ are magnetic field and induction increments when small AC hysteresis loop is superimposed at each point $\left(H_{1}, B_{1}\right)$ of the initial curve $[6,10]$.

\section{Results and discussion}

In the previous work [10], the relation for irreversible relative permeability at initial magnetization curve $\mu_{\mathrm{irr}}^{I N}$ was derived based on the idea of the integration segments and the change of boundaries in the integral of the DC energy losses $W_{D C}$. The latter represent the area of DC hysteresis loop, and is usually obtained by the integration through the values of magnetic field $H$ in a closed cycle along hysteresis loop [13]. In the approach proposed by us, the integration is performed through the positive $H$ values running along the initial curve from 0 to maximum magnetic field $H_{m}$. The elementary contribution $\mathrm{d} W_{\mathrm{DC}}$ becomes the meaning, as illustrated in Fig. 1a, and the expression comprising irreversible relative permeability at hysteresis loop $\mu_{\text {irr }}^{\mathrm{LOOP}}$ is substituted by the expression comprising irreversible relative permeability at initial curve $\mu_{\mathrm{irr}}^{\mathrm{IN}}$ :

$$
\begin{gathered}
W_{\mathrm{DC}}=\oint_{H} H \mu_{0}\left(\mu_{\mathrm{rev}}^{\mathrm{LOOP}}+\mu_{\mathrm{irr}}^{\mathrm{LOOP}}\right) \mathrm{d} H= \\
\int_{0}^{H_{m}} H \mu_{0} F^{-1} \mu_{\mathrm{irr}}^{\mathrm{IN}} \mathrm{d} H,
\end{gathered}
$$

with $F$, generally the function of $H$, introduced in [10]. Form (2), the relation for $\mu_{i r r}^{I N}$ can be obtained

$$
\mu_{\mathrm{irr}}^{\mathrm{IN}}=\frac{F}{\mu_{0} H} \frac{\mathrm{d} W_{\mathrm{DC}}}{\mathrm{d} H} .
$$

This quantity connects the information (i) from an initial curve, where at each its point the irreversible permeability describes the proportion of irreversible magnetization processes, and (ii) from DC hysteresis loop, which area reveals the amount of energy dissipated accompanying irreversible processes within one magnetizing cycle.

In [11] it was found that in the special case of Fe-resin SMCs the typical shapes of the DC hysteresis loops can be approximated by parallelogram and triangles, i.e., by linear functions (Fig. 1b). The linear functions approximation (LFA) enabled to quantify the $W_{\mathrm{DC}}$ with higher accuracy, and covered wider induction range of validity for the case of Fe-resin SMC compared to Steinmetz law $[3,11]$.

The $W_{D C}$ after LDA treatment takes the following form $W_{\mathrm{DC}}=3 H_{C} B=3 H_{C} \mu_{0} \mu_{\text {tot }} H$, where $H_{C}$ is the coercive field, and $\mu_{t o t}$ is the total relative permeability that is defined at initial curve as $\mu_{t o t}=B /\left(\mu_{0} H\right)$. (Notation $(H, B)$ means each point of initial curve which is

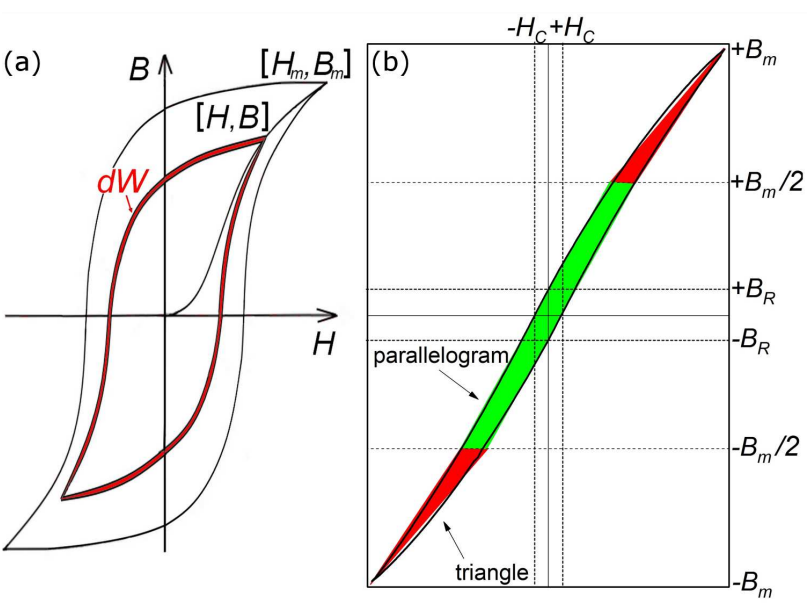

Fig. 1. Illustration of the meaning of elementary contribution to DC losses (on general hysteresis loop) in the derivation of irreversible permeability relation (a), and (b) the linear functions approximation illustrated on typical hysteresis loop of Fe-resin soft magnetic composite.

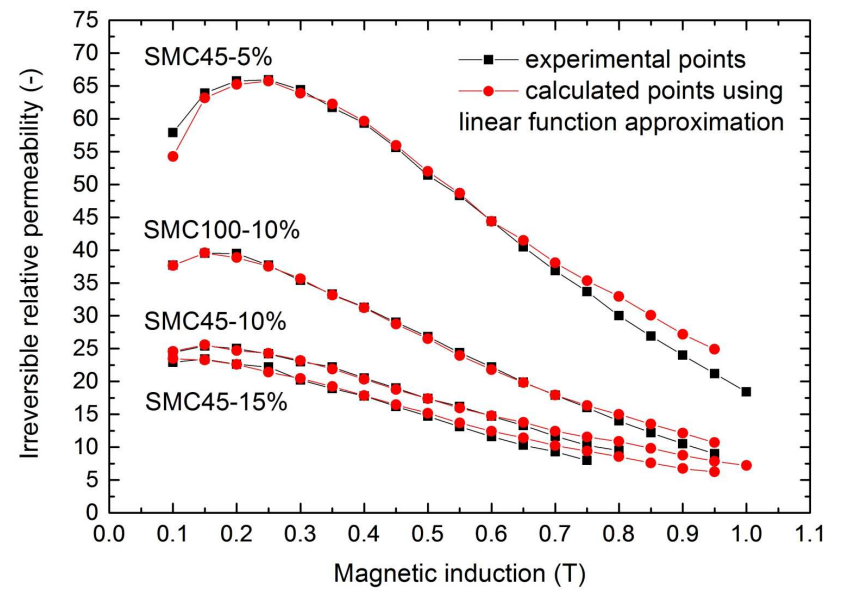

Fig. 2. Comparison of experimental dependencies of irreversible relative permeability at initial curve vs. magnetic induction with the ones calculated using the linear functions approximation for Fe-resin composites.

concurrently the maximum induction point of minor DC hysteresis loop). Applying this derived $W_{D C}$ expression into (3), we obtain the relation for $\mu_{i r r}^{I N}$ specified for Feresin SMCs:

$$
\begin{aligned}
& \mu_{i r r}^{I N}=3 F_{\mathrm{Lin}}^{\mathrm{SMC}} \mu_{\mathrm{tot}} H_{C} / H, \\
& \text { or } \quad \mu_{\mathrm{irr}}^{\mathrm{IN}}=3 F_{\mathrm{Lin}}^{\mathrm{SMC}} \mu_{0} H_{C} \mu_{\text {tot }}^{2} / B,
\end{aligned}
$$

where the function $F$ is denoted as $F_{\text {Lin }}^{\mathrm{SMC}}$. Other parameters $H, B, H_{C}$, and $\mu_{t o t}$, are connected with the minor DC hysteresis loop.

Figure 2 shows a good agreement (relative standard deviation less than $5 \%$ ) between the experimental dependencies of $\mu_{\mathrm{irr}}^{I N}$ on $B$ and those calculated according to LFA, see (4), for samples SMC45-5\%, SMC45-10\%, 
Constant $C_{\mathrm{Lin}}^{\mathrm{SMC}}$ for SMC samples

TABLE II

\begin{tabular}{l|r|r|r|r}
\hline \hline Sample & SMC45-5\% & SMC45-10\% & SMC100-10\% & SMC45-15\% \\
\hline$C$
\end{tabular}

SMC100-10\%, and SMC45-15\%. A wide range of the induction $B \in(0.1,0.8) \mathrm{T}$ has been covered together with one constant value of $F_{\mathrm{Lin}}^{\mathrm{SMC}}=C_{\mathrm{Lin}}^{\mathrm{SMC}}$ in (4), corresponding to the range of validity of LFA [11]. The constant $C_{\mathrm{Lin}}^{\mathrm{SMC}}$ (values are in Table II) are higher for the higher resin content in SMC, and did not depend on Fe particle size. Compared to the previous Steinmetz law based relation for Fe-resin SMCs [10], the new LFA based relation, namely (4), with $F_{\mathrm{Lin}}^{\mathrm{SMC}}=C_{\mathrm{Lin}}^{\mathrm{SMC}}$ is able to cover the wider induction range with higher accuracy.

The correspondence between the two approaches: the LFA approximation of $W_{\mathrm{DC}}$ and the LFA based relation for $\mu_{\mathrm{irr}}^{\mathrm{IN}}$ - is remarkable. Both approaches exhibit the high accuracy, and point at the specific magnetization curves shapes, thus the specific character of magnetization reversal of Fe-resin SMCs compared to majority of ferromagnetic materials.

\section{Conclusion}

The derivation of the relation for irreversible relative permeability at initial magnetization curve $\mu_{\text {irr }}^{\mathrm{IN}}$ was specified using the linear functions approximation (LFA) for Fe-resin soft magnetic composites. These samples differed in content of ferromagnetic filler and particle size distribution. This study is a continuation from a previous work [10]. The LFA expresses the DC energy losses exclusively for Fe-resin composite material as a function of magnetic induction, by approximating the shape of minor hysteresis loop typical for such composites with the parallelogram and triangles, i.e., linear functions. The coercive field $H_{C}$ and the DC energy losses expressions were used in the form $W_{\mathrm{DC}}=3 H_{C} B$, where $B$ denotes the induction of minor hysteresis loop peak point lying on an initial curve. Comparing the experimental and the calculated dependencies of irreversible permeability vs. magnetic induction, it was found that the empirical function is a constant $C_{L i n}^{S M C}$, where the final form of relation is $\mu_{\mathrm{irr}}^{\mathrm{IN}}=3 C_{\mathrm{Lin}}^{\mathrm{SMC}} H_{C} \mu_{0} \mu_{\mathrm{tot}}^{2} / B$. The induction range of validity of the LFA turned out to be wider than that of the Steinmetz law for the special case of Fe-based soft magnetic composites. Importantly, it covers with higher accuracy the same range than the previous one [10] derived from the Steinmetz law. The relation proposed in this paper enables to express the irreversible permeability especially for the Fe-resin soft magnetic composites, in order to determine the proportions of irreversible magnetization processes at each point of initial curve.

\section{Acknowledgments}

This work was realized within the frame of the project "MACOMA" financed by Slovak Research and Development Agency under the contract APVV-15-0115; and by Scientific Grant Agency of Ministry of Education of Slovak Republic and Slovak Academy of Science - projects VEGA 1/0143/20, VEGA 1/0225/20 and VEGA 2/0108/18. This work was also supported by the Development Operational Program Research and Innovation for the project "New unconventional magnetic materials for applications", ITMS: 313011T544, co-funded by the European Regional Development Fund; and by the project "Research Centre of Advanced Materials and Technologies for Recent and Future Applications PROMATECH" ITMS: 26220220186, financed by the Operational Program Research and Development financed through European Regional Development Fund.

\section{References}

[1] K.H.J. Buschow, Concise encyclopedia of magnetic and superconducting materials, Elsevier, Oxford 2005.

[2] K.J. Sunday, M.L. Taheri, Metal Powd. Rep. 72, 6 (2017).

[3] E.A. Périgo, B. Weidenfeller, P. Kollár, J. Füzer, Appl. Phys. Rev. 5, 031301 (2018).

[4] J.-L. Mattei, M. Le Floc'h, J. Magn. Magn. Mater. 257, 335 (2003).

[5] O. Barrière, C. Appino, F. Fiorillo, C. Ragusa, M. Lecrivain, L. Rocchino, H. Ben Ahmed, M. Gabsi, F. Mazaleyrat, M. LoBue, IEEE Trans. Magn. 49, 1318 (2013).

[6] S. Chikazumi, Physics of Ferromagnetism, 2nd ed., Oxford University Press Inc., New York 1999, p. 11.

[7] D.L. Atherton, M. Schonbachler, IEEE Trans. Magn. 24, 616 (1988).

[8] K.S. Ryu, S.H. Nahm, J.S. Park, K.M. Yu, Y.B. Kim, D. Son, J. Magn. Magn. Mater. 251, 196 (2002).

[9] Z. Birčáková, P. Kollár, B. Weidenfeller, J. Füzer, M. Fáberová, R. Bureš, J. Alloys. Comp. 645, 283 (2015).

[10] Z. Birčáková, P. Kollár, J. Füzer, R. Bureš, M. Fáberová, J. Phys. D: Appl. Phys. 51, 395002(10pp) (2018).

[11] P. Kollár, V. Vojtek, Z. Birčáková, J. Füzer, M. Fáberová, R. Bureš, J. Magn. Magn. Mater. 353, 65 (2014).

[12] Höganäs AB Sweden, Polycrystalline Fe powder ASC 100.29, www.hoganas.com.

[13] In Ref. [6] p. 27. 